\title{
Laser Doppler flowmetry signals: pointwise Hölder exponents of experimental signals from young healthy subjects and numerically simulated data
}

\author{
Benjamin Buard $^{1,2}$, Anne Humeau ${ }^{1,2}$, David Rousseau ${ }^{2}$, François Chapeau-Blondeau ${ }^{2}$, \\ and Pierre Abraham ${ }^{3}$
${ }^{1}$ Groupe ESAIP, 18 rue du 8 mai 1945, BP 80022, 49180 Saint Barthélémy d'Anjou cedex, France
${ }^{2}$ Laboratoire d'Ingénierie des Systèmes Automatisés (LISA), Université d'Angers, 62 avenue Notre Dame du Lac, 49000 Angers, France
${ }^{3}$ Laboratoire de Physiologie et d'Explorations Vasculaires, UMR CNRS 6214-INSERM 771,
Centre Hospitalier Universitaire d'Angers, 49033 cedex 01 Angers, France
phone: + 33 (0)2 419665 10, fax: +33 (0)2 419665 11, email: bbuard@esaip.org

\begin{abstract}
We analyze the complexity of laser Doppler flowmetry (LDF) signals which give a peripheral view of the cardiovascular system. For this purpose, experimental and numerically simulated LDF signals are processed. The experimental signals are recorded in young healthy subjects. The numerically simulated LDF data are computed from a model containing six nonlinear coupled oscillators reflecting six almost periodic rhythmic activities present in experimental LDF signals. In the model, the oscillators are coupled with both linear and parametric couplings in order to represent cardiovascular system behaviors. To our knowledge this modeling has never been proposed yet. The complexity of all the experimental and simulated signals is studied by the computation of pointwise Hölder exponents. The latter identify the possible multifractal characteristics of data. The pointwise Hölder exponents are determined with a parametric generalized quadratic variation based estimation method first calibrated from white noise measures. The results of our signal processing analysis show that experimental LDF signals are weakly multifractal for young healthy subjects at rest. Furthermore, our findings together with another recent work of our group show that pointwise Hölder exponents of the simulated data do not describe the ones of the young healthy subjects but are closer to the ones of elderly healthy people. This paper provides useful information to go deeper into the modeling of LDF data, that could bring enlightenment for a better understanding of the peripheral cardiovascular system.
\end{abstract}

Keywords - Laser Doppler flowmetry, multifractality, Hölder exponent, nonlinear oscillator, biomedical engineering

\section{INTRODUCTION}

Laser Doppler flowmetry (LDF) is commonly used in clinical research for monitoring microvascular perfusion. LDF signals are generated by the interaction between photons of a laser light and moving scatterers, mainly red blood cells. Both concentration and velocity of the moving scatterers affect the LDF perfusion estimate [1].
In this paper, we analyze the complexity of LDF signals which give a peripheral view of the cardiovascular system. For this purpose, experimental and numerically simulated LDF signals are processed. The experimental signals are recorded in young healthy subjects. Recent works have shown that LDF signals, recorded in young healthy subjects at rest, are weakly multifractal [2], but that aging can lead to a reduced multifractality [3]. This information is important as it could help in the modeling of the peripheral cardiovascular system: an accurate modeling should behave in the same way as the system it aims to reproduce. For LDF signals, a set of five nonlinear oscillators coupled with linear couplings has recently been proposed as a theoretical model [4]-[6]. We propose herein to numerically simulate, for the first time, LDF signals with six nonlinear oscillators (reflecting six almost periodic rhythmic activities present in experimental LDF signals) coupled with a combination of both linear and parametric couplings (in order to represent cardiovascular system behaviors). To analyze the multifractality of these simulated signals, a computation of their pointwise Hölder exponents is done in comparison with the ones of experimental LDF signals. For the computation of the Hölder exponents, we propose to use the parametric generalized quadratic variation (GQV) based estimation method as the latter, being applied on microvascular data, has proved to give interesting results [2], [4]. Moreover, in order to have a better interpretation of the results, the GQV based estimation method is first calibrated with a measure of white noise.

Our paper is organized as follows: we first introduce the theoretical model of LDF signals. Then, the theory of the GQV method is presented, as well as the tool used for calibration. We then apply the GQV method on simulated and experimental LDF data and present the results that we comment. Finally, we end with a conclusion. 


\section{THEORETICAL MODEL OF LDF SIGNALS}

\section{A. Physiological and theoretical principles}

On the time scale of minutes, six subsystems can be considered to contribute to the regulation of blood flow. Thus, under stationary conditions, when a healthy subject is at physical and mental pause, six characteristic frequencies can be found in LDF signals [7], [8]: $1.1 \mathrm{~Hz}$ for the heart beats, $0.36 \mathrm{~Hz}$ for the respiration, $0.1 \mathrm{~Hz}$ for the myogenic activity, $0.04 \mathrm{~Hz}$ for the neurogenic activity, $0.01 \mathrm{~Hz}$ for the endothelial-related metabolic activity, and $0.007 \mathrm{~Hz}$ for endothelium mechanisms, such as endothelium-derived hyperpolarizing factor (EDHF). The last one has recently been found [8]. The characteristic frequency values are different from subject to subject, but are found in the same frequency intervals for all subjects.

As a result of mutual couplings between the subsystems, Ref. [4] has shown that the values of the characteristic frequencies are time-variable and that their corresponding amplitudes are modulated. Based on these findings, some authors proposed to simulate LDF signals with five nonlinear coupled oscillators [4]-[6]. To take into account the last discoveries, a sixth oscillator has been added in our simulation. The basic unit in the model is written as [4]-[6]:

$$
\begin{aligned}
& \dot{x}_{i}=-x_{i} q_{i}-\omega_{i} y_{i}+g_{x_{i}}(x) \\
& \dot{y}_{i}=-y_{i} q_{i}+\omega_{i} x_{i}+g_{y_{i}}(y) \\
& \text { with } q_{i}=\left(\sqrt{x_{i}^{2}+y_{i}^{2}}-a_{i}\right) \times \alpha_{i}
\end{aligned}
$$

where $\mathbf{x}$ and $\mathbf{y}$ are vectors of oscillators state variables, $\alpha_{i}$, $a_{i}$, and $\omega_{i}$ are constants, $\mathbf{g}_{\mathbf{x}_{\mathbf{i}}}(\mathbf{x})$ and $\mathbf{g}_{\mathbf{y}_{\mathbf{i}}}(\mathbf{y})$ are coupling vectors. Some couplings have been proposed [6], and it has been suggested that both linear and parametric couplings have to be taken into account. That is why, for the first time, mixed couplings (linear and parametric) are introduced herein in the simulated signals.

For the rhythmic cardiac activity the model is:

$$
\begin{aligned}
\dot{x}_{1}= & -x_{1} q_{1}+\eta_{2} x_{2}-\eta_{3} x_{3}-\eta_{4} x_{4}+\eta_{5} x_{5}+\eta_{6} x_{6} \\
& -y_{1}\left(\omega_{1}+\eta_{2} x_{2}-\eta_{3} x_{3}-\eta_{4} x_{4}+\eta_{5} x_{5}+\eta_{6} x_{6}\right)
\end{aligned}
$$

A similar equation is used for the vector $\dot{y}_{1}$.

For the five other subsystems, the modeling, based on Ref. [6], is in the same form.

\section{B. Adjustment of the model}

In the present work, the above-mentioned model is numerically simulated. Its adjustment (choice of the constant

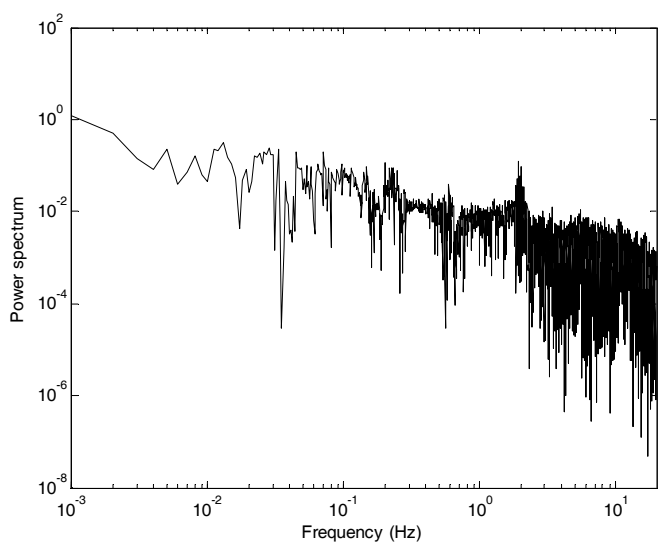

(a)

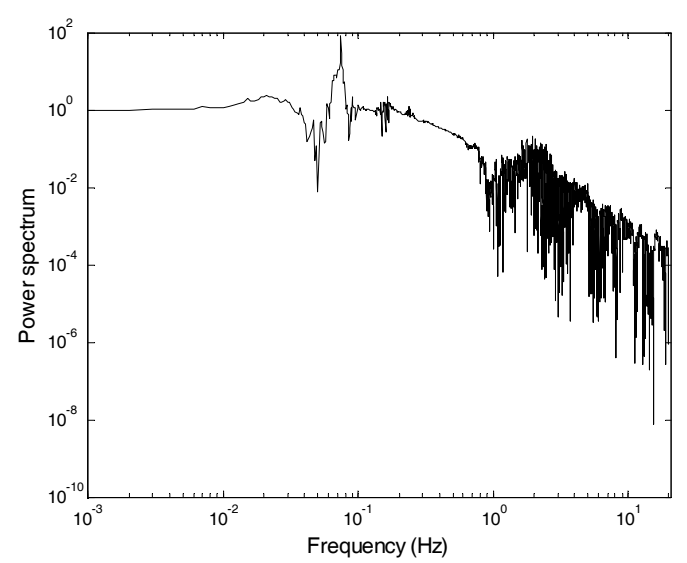

(b)

Figure 1 (a) Power spectrum of an experimental LDF signal recorded in a young healthy subject at rest. (b) Power spectrum of a simulated signal $\left(0.6 * x_{1}+0.0002 * x_{2}+0.6 * x_{3}+0.2 * x_{4}+0.6 * x_{5}+0.5 * x_{6}\right)$.

parameters) is done from the frequency domain: the constant parameters of the model are chosen so that the power spectrum of the simulated signal is close to the power spectrum of experimental LDF signals (see Fig. 1). The experimental signals have been recorded on seven young healthy men (between 20 and 36 years) with a laser Doppler flowmeter (Periflux 5000, Perimed AB, Stockholm, Sweden) and a probe positioned on the forearm. A representative example is shown in Fig. 1.

\section{MultifRactality ANALYSIS}

In our work we use the parametric GQV based estimation method to compute Hölder exponents of experimental and simulated LDF signals. Moreover, in what follows a calibration of the method is proposed. 


\section{A. GQV method}

The parametric GQV based estimation method [9] is used to compute Hölder exponents of signals. For one dimensional multifractional Brownian motion $(\mathrm{mBm})$, a simple algorithm to estimate the Hölder exponent $H\left(t_{i}\right)$ of a $\mathrm{mBm} \widetilde{B}$, sampled at the moments $i / N, i=0, \ldots, N-1$ can be used [10].

In the case of an $\mathrm{mBm}$ non-normalized, a squared mean linear regression using the propriety of local autosimilarity of the $\mathrm{mBm}$ is usually proposed. We thus get [10]:

$$
H\left(t_{i}\right)=-\frac{\beta_{1}\left(t_{i}\right)-(1-\gamma)}{2 \delta}
$$

where $\gamma$ and $\delta$ are parameters such as $\delta-\gamma>1 / 2$ and $\gamma \geq \delta$, and $\beta_{1}$ is the slope (see more in Ref. [10]). [10]:

To correct the noise, an adjustment has been proposed

$$
H_{\text {max }_{-} \text {reg }}=H_{\text {max }}-\bar{H}_{\text {max }}+\bar{H}_{\text {reg }}
$$

where $H_{\max }$ is the estimate obtained without regression and with $N=N_{\max }$, and $H_{\text {reg }}$ is the estimate obtained with regression. Good results have been obtained with this algorithm [10]. In our work, the parametric GQV method is carried out with the Fraclab v2.0 tool [11].

\section{B. Calibration of the $G Q V$ based estimation method}

In order to calibrate the parametric GQV based estimation method for its application on LDF data, we first apply it on a white noise measure as well as on its first and second order linearly filtered versions. White noises and their first order linearly filtered versions are known to be not differentiable (too irregular). We have to go up to a second order linear filter to obtain differentiable signals [12]. These characteristics are therefore used herein in order to evaluate the parametric GQV based estimation method.

The results are presented in Fig. 2. Hölder exponents lower than one mean that the corresponding signal is not differentiable. On the contrary, a signal with Hölder exponents higher than one is differentiable. The results obtained with the parametric GQV based estimation method concerning a white noise realization and its filtered versions are thus qualitatively in accordance with the theory. From these first results, the parametric GQV based estimation method can be applied in its present form (FracLab v2.0 tool [11]) on experimental and simulated LDF signals.

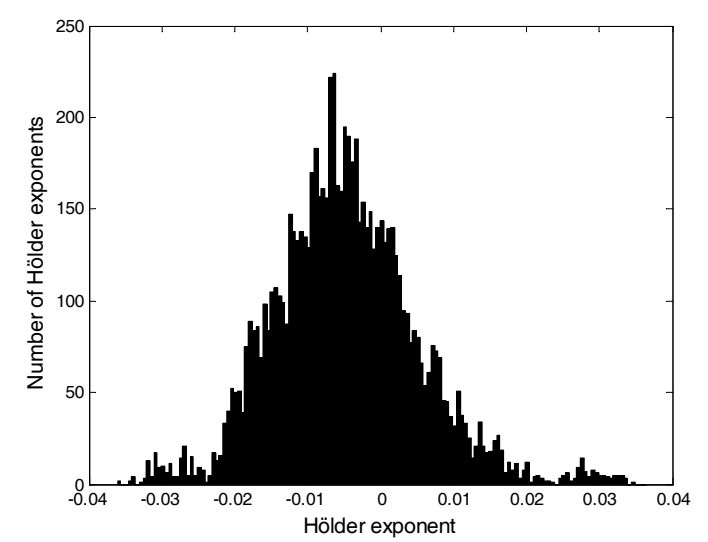

(a)

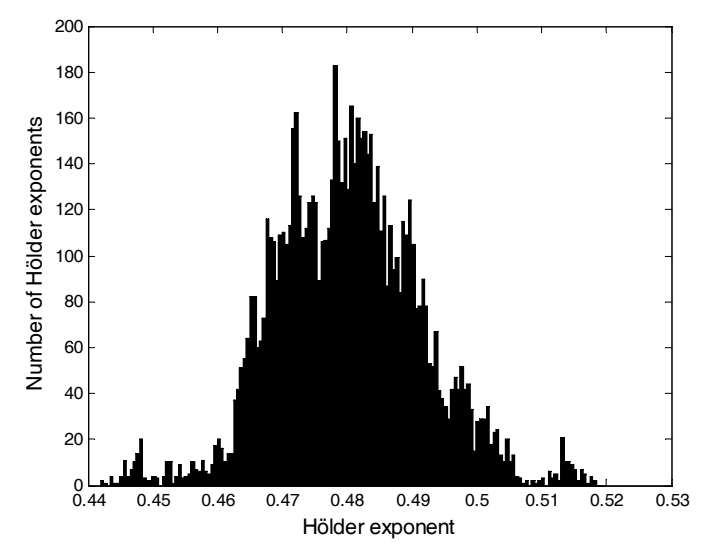

(b)

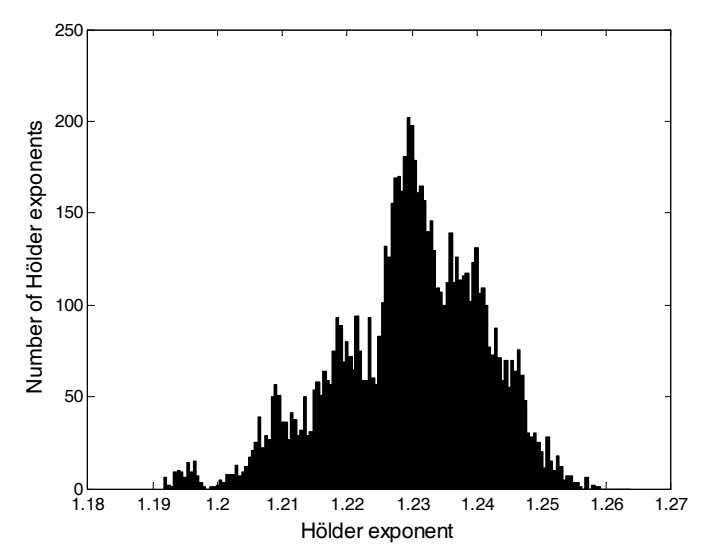

(c)

Figure 2 Histogram of Hölder exponents, obtained with the parametric GQV based estimation method for (a) a white noise measure,

(b) its first order linear filtered version, and (c) its second order linear filtered version. 


\section{HÖLDER EXPONENTS OF LDF SIGNALS}

We first compute the Hölder exponents of the experimental LDF signals. For the representative example presented in Fig. 3, the Hölder exponents are between 0.5379 and 0.6582 (width of 0.1203 ). The other experimental signals have Hölder exponents in the same range. Hölder exponents of our adjusted simulated signal (see Fig. 4) vary from 1.2518 to 1.3497 (width of 0.0979 ).

Our work therefore shows that the widths of Hölder exponents from LDF signals, experimental and simulated, are quite similar and relatively small. We can infer that LDF signals (experimental and simulated) are weakly multifractal. Moreover, the simulated signal is differentiable contrary to the experimental signals. Furthermore, it has been shown that Hölder exponents of LDF signals increase with age and

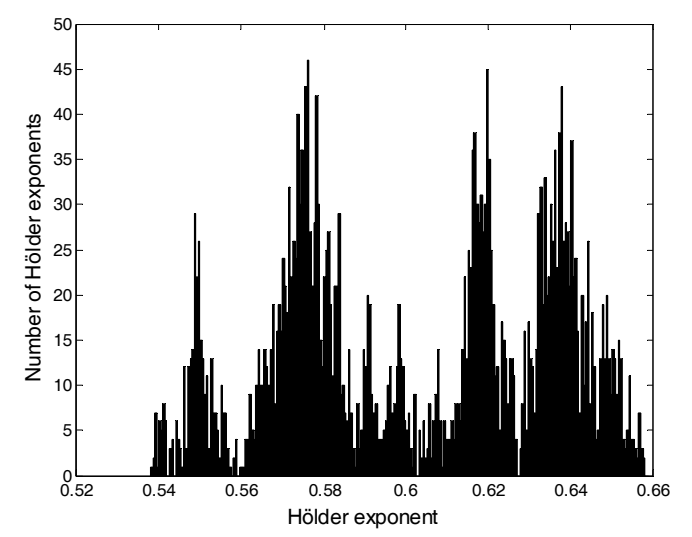

Figure 3 Histogram of Hölder exponents, obtained with the parametric GQV based estimation method for an experimental LDF signal.

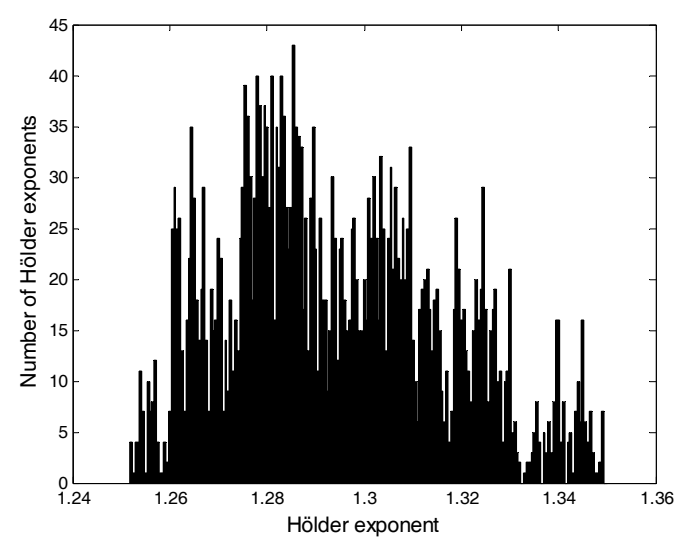

Figure 4 Histogram of Hölder exponents, obtained with the parametric GQV based estimation method for a simulated signal. can become higher than one [3]. We can therefore deduce that the model used can better describe the complexity of LDF signals from elderly subjects rather than from young healthy subjects.

\section{CONCLUSION}

Our study confirms a weak multifractal behavior for peripheral blood flow signals, for young healthy subjects at rest. It contributes to a quantitative assessment of the complexity of the data recorded from peripheral locations where intricate interactions at the microcirculation level take place. Moreover, first, the comparison between the values of the Hölder exponents of simulated and experimental signals leads to the conclusion that the model of six oscillators using linear and parametric couplings is not adequate to reproduce the multifractal behavior observed in young healthy subject. Secondly, our results show that the model would be more accurate to reproduce the multifractal behavior observed in elderly subjects.

This paper provides useful information to go deeper into the modeling of LDF data, and bring information for a better understanding of the peripheral cardiovascular system. Our results may therefore offer some guidelines for the construction of more adaptable models of LDF signals that could provide relevant physiological information.

\section{AcKNowledgements}

B. Buard acknowledges support from La Région des Pays de la Loire, France.

\section{REFERENCES}

[1] Nilsson G E (1984) Signal processor for laser Doppler tissue flowmeters. Med. Biol. Eng. Comput, 22:343-348

[2] Humeau A, Chapeau-Blondeau F, Rousseau D, Tartas M, Fromy B, Abraham P (2007) Multifractality in the peripheral cardiovascular system from pointwise Hölder exponents of laser Doppler flowmetry signals. Biophys. J. 93:L59-L61

[3] Humeau A, Chapeau-Blondeau F, Rousseau D, Trzepizur W, Abraham P (2008) Multifractality, sample entropy, and wavelet analyses for age- related changes in the peripheral cardiovascular system: preliminary results. Med. Phys. 35:717-723

[4] Stefanovska A, Strle S, Bracic M, Haken H (1999) Model synthesis of the coupled oscillators which regulate human blood flow dynamics Nonlin. Phenom. Complex Syst. 22:72-87

[5] Stefanosvka A, Bracic Lotric M, Strle S, Haken H (2001) The cardiovascular system as coupled oscillators? Physiol. Meas. 22:535-550

[6] Stefanovska A, Luchinsky D G, McClintock PVE (2001) Modelling couplings among the oscillators of the cardiovascular system. Physiol. Meas. 22:551-564 
[7] Stefanovska A, Bracic M, Kvernmo H D (1999) Wavelet analysis of oscillations in the peripheral blood circulation measured by laser Doppler technique. IEEE Trans. Biomed. Eng. 46:1230-1239

[8] Kvandal P, Landsverk S A, Bernjak A, Stefanovska A, Kvernmo H D, Kirkebøen K-A (2006) Low-frequency oscillations of the laser Doppler perfusion signal in human skin. Microvasc. Res. 72:120-127

[9] Istas J, Lang G, (1997) Quadratic variations and estimation of the local Hölder index of a Gaussian process. Annales de l'Institut Henri Poincaré (B) Probabilités et Statistiques 33:407-436
[10] Barrière O (2007) Synthèse et estimation de mouvements Browniens multifractionnaires et autres processus à régularité prescrite. Définition du processus auto régulé multifractionnaire et applications. $\mathrm{PhD}$ thesis, Ecole Centrale de Nantes, and Université de Nantes, France

[11] Lévy Véhel J, Legrand P (2004) Signal and Image processing with FracLab. FRACTAL04, Complexity and Fractals in Nature, $8^{\text {th }}$ International Multidisciplinary Conference, Vancouver, Canada

[12] Papoulis A (1991) Probability, Random Variables, and Stochastic Processes. McGraw-Hill, New York 